\title{
Geografia Distribuo de Tertremaj Domaĝoj kun Speciala Referenco al la Efikoj de la Nankaido Tertremo, la 21-an de Decembro, 1946, en Koti Prefektejo, Sikoku, Japanujo.*
}

\author{
De \\ Setumi Miyamura, \\ Instituto por Esploroj de Tertremo, Universitato de Toleio, \\ Yatuka SAIZYo, \\ Kemia Instituto, Tolkio Toritu Daigalcu,** \\ $\mathrm{kaj}$ \\ Sōhei KaIzuka, \\ Geografia Instituto, Tolio Toritu Daiga/eu.**
}

\begin{abstract}
Geographical Distribution of Earthquake Damages with Special Reference to the Effects of the Nankaido Earthquake, Dec. 21, 1946, in Kochi Prefecture, Shikoku, Japan.
\end{abstract}

Concerning the relations between geomorphological ground conditions and earthquake damages throughout the Prefecture of Kochi, Shikoku, Japan, due to the Great Nankaido Earthquake of December 21st, 1946, we have noticed several interesting facts:

i) On the sandy ground of beach ridges, damages were found only in those parts, where the basement bed rock lies deep, or where some kind of soft mud layers lies beneath sand, or where the ground consists of very soft sand, which has been deposited in very recent years or accumulated by wind blow. On the contrary, there was scarcely any damage, where the bed rock lies near the surface, or where hard materials such as pebbles or stones lie under the sandy ground, or where the surface sand are consolidated. Practically, of course, some of the above mentioned conditions coexist complicatedly combined in the same place.

ii) There were severe damages on swampy low lands behind the beach ridge.

iii) Even on alluvial plains, we found patches of rather slight damages among otherwise severe damages. Under those patches of slight damages stony layers were usually found as is the case on beach ridges.

We have examined the hardness of the surface ground by a simple hand-boring stick of about $1 \mathrm{~m}$. length.

Existence of the stony layers under the ground was conjectured by the distribution of Cl' contents in underground water. Where there are stony layers under the ground, underground water flows constantly and the Cl' contents must be small. On the contrary, underground water cannot flow easily in clay, mud or fine sand layers, and the $\mathrm{Cl}$ ' contents must become great there. A typical example of the above two cases was encountered in the town of Nakamura, where the geographical distribution of earthquake damages clearly agrees with the Cl' contents distributions. (Fig. 10)

* International language EsPeranto.

** Tokio Toritu Daigaku signifas Ĉefurba Universitato de Tokio aŭ Metropolitan University of Tokyo. La junaj aŭtoroj apartenis ĝis 1949 al la Geografia Instituto, Naturscienca Fakultato, Universitato de Tokio. 


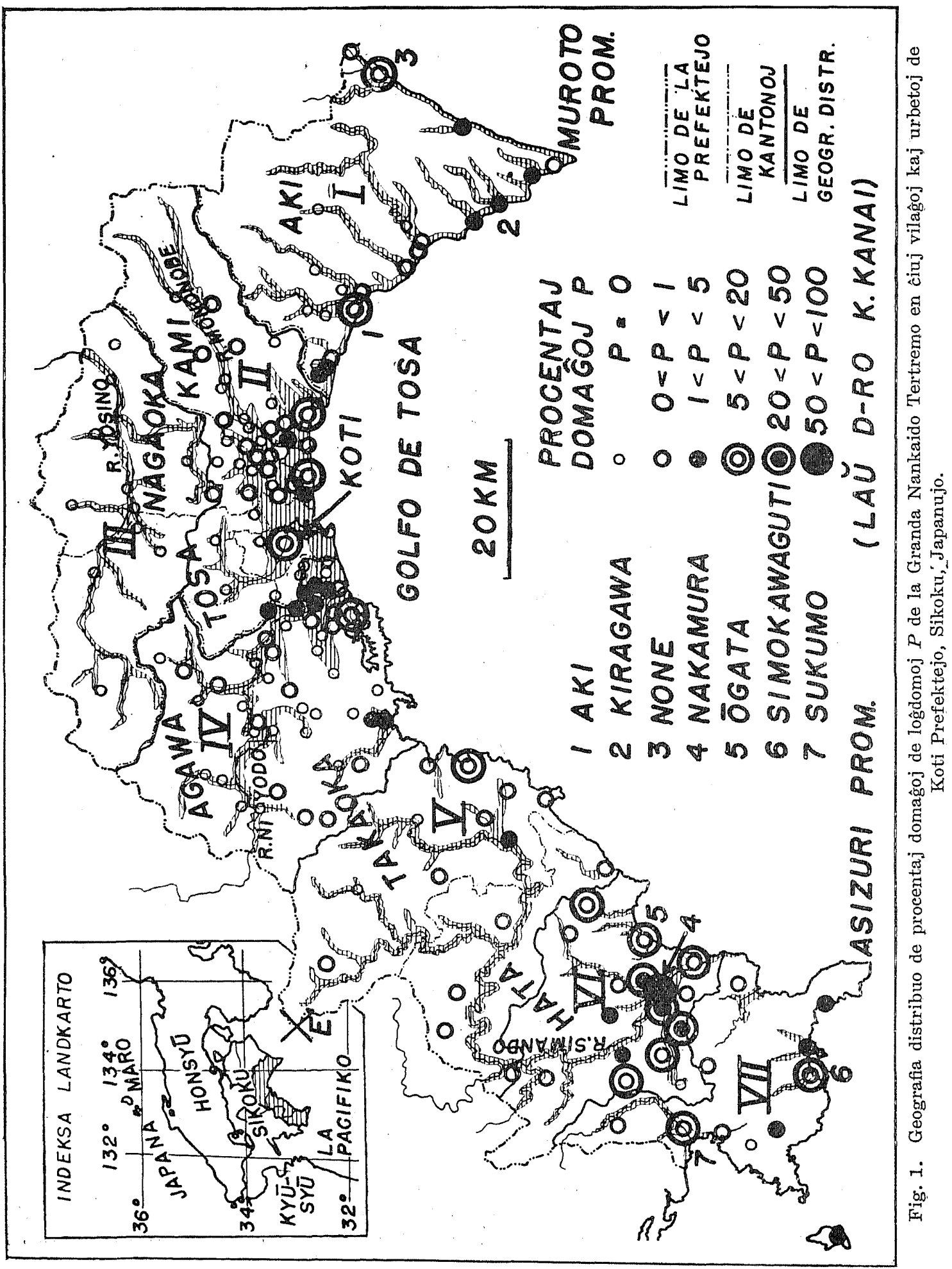


De la granda tertremo de 21 -a de decembro, 1946, kiu generale estas nomata Nankaidoaŭ Nankai-Tertremo, la plej severaj domấgoj estis suferataj en la suda parto de Sikoku Insulo, nome en la Prefektejo Koti. La epicentro de ci tiu tertremo estis proksimume ĉe $135^{\circ} \mathrm{E}, 33^{\circ} \mathrm{N}$ laŭ provizora publikigo de la Centra Meteorologia Observatorio. Sed la domaĝoj de la tertremo montris tre komplikitan distribuon en ćiuj partoj de la sudokcidenta Japanujo laŭ la diversaj naturoj de grundoj kaj aliaj kondiĉoj, senclepende de epicentra distanco. Ekzemple, la urbeto Nakamura kaj ĝia cirkaŭajo en la kantono Hata, Koti-Prefektejo, suferis la plej grandajn domaĝojn de ci tiu tertremo, kvankam la loko situacias cirkaŭ $200 \mathrm{~km}$ malproksime de la epicentro.

Pri la domaĝoj de la tertremo en KotiPrefektejo, esploroj de diversaj vidpunktoj kaj diversaj metodoj estis jam faritaj de kelkaj kolegoj en nia instituto, nome, de d-roj K. Kanar, H. Kawasumi, T. Minakami, S. SAkuma, Y. Sato kaj aliaj. La nunaj aŭtoroj esploris la temon precipe pri interrilatoj de tertremaj domaĝoj kaj naturoj de grundoj de la vidpunktoj de geomorfologio, hidrologio kaj geologio. Nu sekvos sube la ĝenerala aspekto de la rezulto de niaj esploroj pri ciu geomorfologia distrikto aparte, kiu estas montrata en Fig. 1.

\section{Distrikto de Muroto-duoninsulo.}

La tuta distrikto apartenas administre al la kantono Aki kaj ĝia tereno estas plejparte montaro kun mallarĝaj valoj. Sekve vilaĝoj kaj urbetoj tie situacias generale sur la marbordo kiel strat-vilaĝoj, escepte de kelkaj, kiuj trovighas sur la roka grundo de la montflanko de valoj en interna lando. Lokoj de urbetoj kaj vilaĝoj estas klasifikataj laŭ la naturoj de grundo kiel sube:

a) roka grundo, b) rivera teraso, c) marborda teraso, d) malalta perono de marerozio, e) marborda digo, f) cirkonferenco de aluvio g) interna parto de aluvio, h) aluvio cirkaŭ river-enfluejo.

Tamen plejmulto da vilaĝoj kaj urbetoj situacias sur la grundoj a), d) kaj e). Sur la grundoj a) kaj d) oni trovis neniajn domaĝojn de la tertremo, kaj sur e) trovigis konsider. indaj domaĝoj en kelkaj partoj. Sur la grundo f) estis malgrandaj domaĝoj kaj sur g) kaj h) troviĝis iom grandaj domaĝoj.

Tamen cie la grado de la tertremaj domaĝoj estis tre komplikitaj laŭ la aliaj diversaj kondicoj de lokoj.

Kiel supre skribite, riveroj en la distrikto havas tre mallarĝajn valojn kaj formas nur malgrandajn aluviajn ebenejojn ce siaj busoj al maro. 'lial la dikecoj de tiuj aluvioj estas malgrandaj kaj, en konsekvenco, oni ne trovas tıe cıel grandajn tertremajn clomaĝojn, kiel oni generale povas trovi sur tiel-nomata aluvia ebenejo. Precipe, ju plı suden oni iras des pli granda oni crovas la lastatempan levighon de grundo, la terasojn altaj, kaj levigintan peronon de marerozio kreskanta, kaj konsekvence, kvankam la aluviaj ebenejoj ce river-enfluejoj estas sufice larĝaj, la aluvio estas tre maldika kaj sur rivertundo malkovrighas roka grundo, ekzemple en la valo de Huttu, en la urbeto Muroto. Tertremaj domaĝjoj estas sendube tre malgrandegaj tie. En la norda parto, la marbordo transiĝas al malleviga tipo de levigha tipo, kaj pli norde de cirkaŭ Aki la riverbuŝaj ebenejoj estas iomete pli larĝa kaj ankaŭ iliaj aluviaj tavoloj estas pli dikaj. Sekve troviĝas iom da domaĝoj en vilaĝoj kaj urbetoj sur cieaj aluvioj. Laŭuonge de la marbordo kreskas sablejo ĉie, krom tie, kie la marbordo estas borderita de levighinta perono de mar-erozio. Kreskado de la sablejo estas tre rimarkinda en la norda parto de la distrikto, kaj laŭlonge de la marbordo de riverbusa aluvio-ebenejo kreskas bekforma aŭ digforma sablaĵ, malantaŭ kiuj estas malaltaj kaj malsekaj terenoj. Marborda sabldigo formas du aŭ tri paralelajn vicojn respondante al siaj nombroj de fojoj de nekontinuaj leviĝoj en la pasintaj jaroj.

Sub la sablejo, kiu borderas la terasojn proksimighantajn al la maro aŭ montojn, estas roka bazo malprofunda kaj la tertremaj domaĝoj tie estas ne tre severaj. Kontraŭe la sablejo, kiu borderas la riverbuŝajn aluvioebenejojn, estas bekforma sablajo ĉe golfenirejo aŭ sablodigo en bordmalproksimeco 
antaŭ la aluvioj estis enterigitaj kaj sekve la dikeco de sab'ejo estas granda kaj en kelkaj partoj sur la tiaj sablejoj trovigîs sufice grandaj domaĝoj de la tertremo. Nome, kiel oni renkontas en la urbetoj Aki, Kiragawa kaj None, severe suferintaj partoj en la urbetoj lokighas $\hat{g}$ enerale aŭ sur sablotavolo pli nova ol aliaj partoj aŭ sur sablejoj, sub kiuj roka bazo estas malprofunđa, kaj precipe tiaj partoj, kiaj konsistas el blovita tavolo de sablejo sur la malseka tereno malantaŭ la marborda digo aŭ sablajo, suferis tre grandajn domaĝjojn de la tertremo. Tio sajnas kompreneble ne kaŭzita de tio, ke en tiuj partoj speciale amasighas malbonaj domoj. (Komparu kazojn de Aki kaj None.)

Sube ni priskribos la rezultojn de la esploroj en kelkaj lokoj de la distrikto.

(1) Urbeto Aki. La urbeto kuŝas laŭlonge sur du vicoj de sablaj digoj, el kiuj norda estas iomete pli alta, $\mathrm{kaj}$ la topografiaj konturlinioj en la urbeto montriĝas en Fig. 2. (Ankaŭ vidu Fig. 1.)

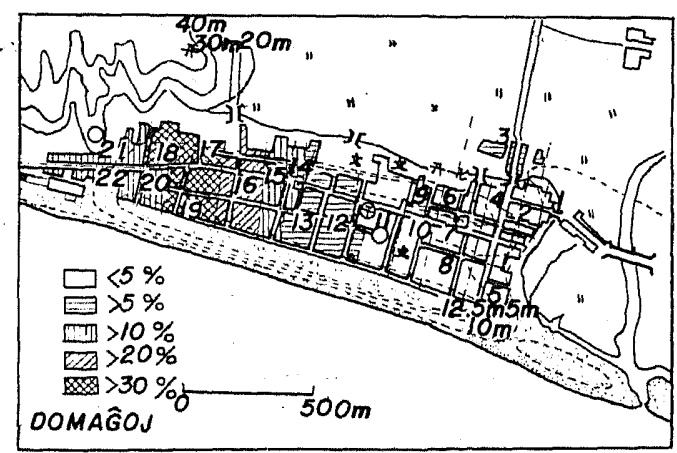

Fig. 2. Domaĝoj de la Nankaido Tertremo en la urbeto Aki, Koti Prefektejo.

De nordo al oriento fiuas unu rivereto, kontraŭ kiun malaltiĝas la tereno kaj ankaŭ en la nord-okcidento la tereno estas malalta, tuŝante al la malantaŭa terciara montaro. Tiu sablejo estis verŝajne bord-malproksima sablajo. Gila meza parto estas la plej mainova, dum la ambaŭ ekstremoj estas pli novaj, kaj el la du vicoj la norda estas sendube pli malnova. Tamen, la suprajo de la norda sabl-digo sajnas formita de blovado.

Laŭ la diroj de putfosistoj, subtera tavolo en norda parto estas speco de marĉa tero kaj sub la sablejo de la orienta parto kusas riverŝtonetoj, dum alie estas nur sufiĉe dika tavolo de sablo.

$\mathrm{Ni}$ ekzamenis la distribuon de $\mathrm{Cl}^{\prime}$-enhavo de la subtera akvo en la urbeto. Generale la distribuo de $\mathrm{Cl}^{\prime}$-enhavo en subtera akvo proksima de marbordo estas paralela al la marborda linio, kiel montrate de la esploro de d-ro Sinkicî Yoshimura, en Simoda, Sizuoka Prefektejo, sed ĉi tie tiela rilato tute ne ekzistas. Krom marborda regiono $\mathrm{Cl}^{\prime}$-distribuo estas ĝenerale superregata de influoj de sterko en la kampo kaj higiena stato en domaro, kaj tio estis videbla ankaŭ ĉi tie. Plie, ĉar ni scias, ke la $\mathrm{Cl}^{\prime}$-enhavo estas multe influata de movado de subterakvo, ni povas konjekti per tio, ke la akvoportanta tavolo sub la tereno estas marĉa aŭ śtonetplena terajo. Generale en marĉa tavolo la subterakvo staras senmova kaj kontraŭe ĝ $\mathrm{i}$ fluadas en stonetplena tavolo. $\mathrm{La}$ distribuo de $\mathrm{Cl}^{\prime}$-enhavo en la urbeto Aki estas montrata en Fig. 3 kun nombroj de gram-valoloj por litro. Kvankam la influo de

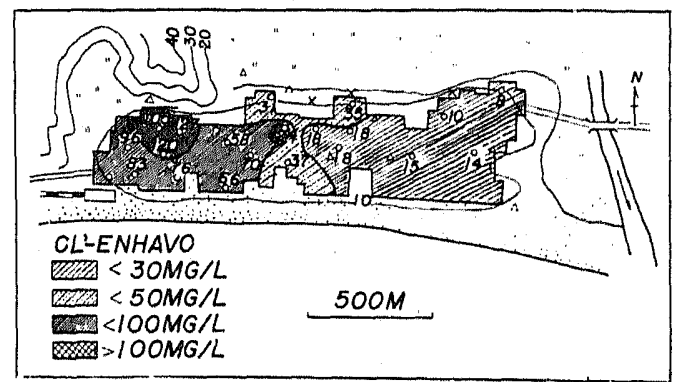

Fig. 3. Cl'-enhavo en subterakvo kaj rezulto de borbastona ekzameno en la urbeto Aki, Koti Prefektejo.

( $\times$ mola sablajo $\Delta$ malmola sablajo)

kampo en norda cikaŭajo kaj de malpura distrikto konsistanta el ŝvarmo de domaĉoj en nord-okcidenta parto estas ambaŭ iomete klara, la plej granda tendenco, kiu duigas la urbeton en partojn okcidentan kaj orientan, estas tio, ke en la orienta parto la subtera tavolo konsistas el riverŝtonetoj kaj sekve tie fluadas pura subterakvo kun malmulta $\mathrm{Cl}^{\prime}$ enhavo, kaj en la okcidenta parto la subtera teraĵo estas ślimo, argilo, aŭ sablo havanta malpuran kaj Cl'-riçan subterakvon. Kompreneble en la orienta parto trovighas ankaŭ 
Tabelo I. Domaĝoj en la urbeto Aki, Koti Prefektejo.

\begin{tabular}{|c|c|c|c|c|c|c|c|c|c|}
\hline \multirow{4}{*}{ No. } & \multirow{4}{*}{ Suburbeto } & \multicolumn{4}{|c|}{ Loĝgdomoj } & \multicolumn{4}{|c|}{ Tenejoj, Staloj, k.t.p. } \\
\hline & & \multicolumn{3}{|c|}{ Nombroj de } & \multirow{3}{*}{$\begin{array}{c}\text { Pro- } \\
\text { centaj } \\
\text { Doma-- } \\
\text { goj } P\end{array}$} & \multicolumn{3}{|c|}{ Nombroj de } & \multirow{3}{*}{$\begin{array}{l}\text { Pro- } \\
\text { centaj } \\
\text { Doma- } \\
\text { goj } P\end{array}$} \\
\hline & & \multicolumn{2}{|c|}{ Detruita j } & \multirow{2}{*}{$\underset{N}{\text { Tutaj }}$} & & \multicolumn{2}{|c|}{ Detruitaj } & \multirow[b]{2}{*}{$\underset{N}{\text { Tutaj }}$} & \\
\hline & & $\begin{array}{l}\text { Tute } \\
N_{g}\end{array}$ & $\begin{array}{c}\text { Duone } \\
N_{h}\end{array}$ & & & $\begin{array}{l}\text { Tute } \\
N_{g}\end{array}$ & $\begin{array}{c}\text { Duone } \\
N_{h}\end{array}$ & & \\
\hline 1 & $1-\mathrm{ku}$ & 1 & 6 & 137 & 2.9 & 1 & & & 0.7 \\
\hline 2 & $2-k u$ & & 3 & 106 & 1.4 & 2 & 1 & & 2.4 \\
\hline 3 & $3-k u$ & 4 & 6 & 115 & 6.1 & & & & 0.0 \\
\hline 4. & $4-\mathrm{ku}$ & 1 & 5 & 95 & 3.8 & 1 & 2 & & 2.1 \\
\hline 5 & $5-\mathrm{ku}$ & 1 & 2 & 82 & 2.4 & 1 & 2 & & 2.4 \\
\hline 6 & $6-\mathrm{ku}$ & 1 & 2 & 80 & 2.6 & & & & 0.0 \\
\hline 7 & $7-\mathrm{ku}$ & & & 66 & 0.0 & & 2 & & 1.5 \\
\hline 8 & $8-\mathrm{ku}$ & & 1 & 126 & 4.4 & 6 & 6 & & 7.1 \\
\hline 9 & 9-ku & & & 109 & 0.0 & & & & 0.0 \\
\hline 10 & $11-\mathrm{ku}$ & 1 & & 128 & 0.8 & 2 & & & 1.6 \\
\hline 11 & $12-\mathrm{ku}$ & & 3 & 127 & 1.2 & $\overline{1}$ & 1 & & 1.2 \\
\hline 12 & $13-\mathrm{ku}$ & 3 & 8 & 104 & 6.8 & 4. & 2 & & 4.8 \\
\hline 13 & 14-ku & 5 & 6 & 104 & 7.7 & 23 & 10 & & 26.9 \\
\hline 14 & $15-\mathrm{ku}$ & 2 & 10 & 70 & 10.1 & & & & 0.0 \\
\hline 15 & $16-\mathrm{ku}$ & 10 & 13 & 84 & 19.7 & 13 & 11 & & 22.0 \\
\hline 16 & $17-\mathrm{ku}$ & 18 & 28 & 88 & 27.3 & 4 & 1 & & 5.1 \\
\hline 17 & $18-\mathrm{ku}$ & 8 & 47 & 96 & 32.8 & 3 & & & 3.1 \\
\hline 18 & $19-\mathrm{ku}$ & 14. & 45 & 122 & 30.0 & 11 & 15 & & 15.2 \\
\hline 19 & $20-\mathrm{ku}$ & 13 & 104 & 196 & 33.2 & 10 & 8 & & 7.1 \\
\hline 20 & $21-\mathrm{ku}$ & 6 & 20 & 163 & 9.9 & 1 & 2 & & 1.2 \\
\hline 21 & $22-\mathrm{ku}$ & 2 & 47 & 102 & 15.7 & 2 & 6 & & 3.1 \\
\hline 22 & $23-k u$ & 1 & 45 & 211 & 11.2 & & & & 0.0 \\
\hline 23 & 24-ku & & & 140 & 0.0 & & & & 0.0 \\
\hline 24 & 25-ku & & 2 & 84 & 1.2 & & 15 & & 8.9 \\
\hline 25 & Anauti & & 3 & 305 & 0.4 & & & & 0.0 \\
\hline & Sumo & 83 & 406 . & 3160 & 9.1 & 85 & 84 & & 4.0 \\
\hline
\end{tabular}

N.B. $P=\left\{\left(N_{g}{ }^{\prime}+N_{h} 12\right) / N\right\} \times 100 \%$. $P$ de tenejoj k.t.p. estas kalkulata, kun supozo ke $N$ estas sama kun tio de loĝdomoj.

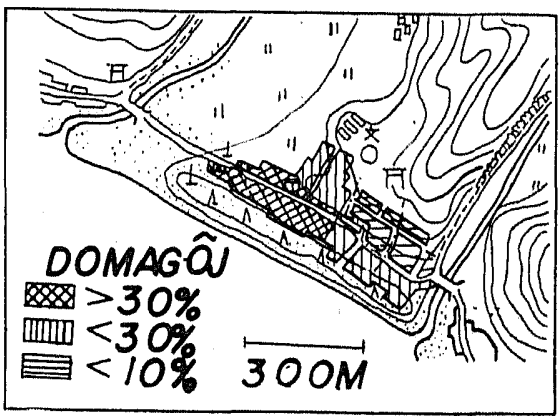

Fig. 4. Procentaj domaghoj $P$ de la Nankaido Tertremo en la urbeto Kiragawa.

domaro de malpura stato!

Por ekzameni solidecon de plej supra terajo, ni uzis borbastonon (kies longeco kaj diametro estas c. $1.5 \mathrm{~m} \mathrm{kaj} 2 \mathrm{~cm}$ respektive) kun stalpinto enŝovante $\hat{g}$ in en la teron. La rezultoj de borbaston-ekzameno en la urbeto montriĝas en Fig. 3 kun la signoj $\times$ kaj $\Delta$, kiuj montras molajn kaj malmolajn lokojn

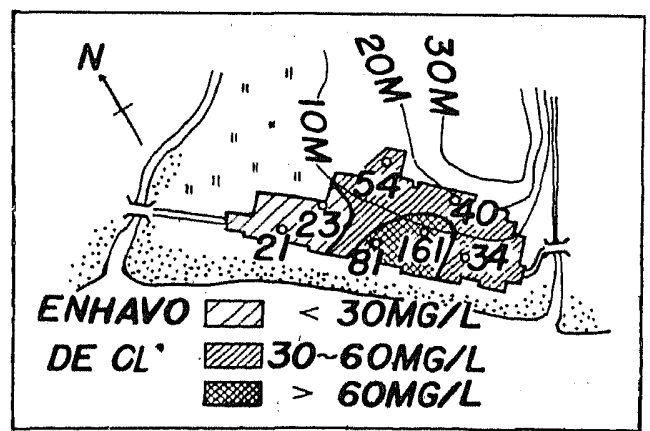

Fig. 5. Cl'enharo en la subtera akro en la vilaĝo de Kiragawa, Koti Prefektejo.

respektive.

Domaj domaĝoj de la lasta granda Nankaitertremo en ciuj kvartaloj (suburbetoj) de la urbeto Aki estas montrata en Tabelo I kaj Fig. 2. La domaĝjoj estas ĝenerale severaj sur nova kaj mola tereno, kaj en la orienta parto, sub kie kuŝas riverŝtonetoj, troviĝas nenia granda domaĝo. En la orienta ekstremo 
Tabelo II. Domaĝoj en la urbeto Kiragawa.

\begin{tabular}{|c|c|c|c|c|c|c|c|c|c|}
\hline \multirow{4}{*}{ No. } & \multirow{4}{*}{ Suburbeto } & \multicolumn{4}{|c|}{ Loĝ̉omoj } & \multicolumn{4}{|c|}{ Tenejoj, Staloj, k.t.p. } \\
\hline & & \multicolumn{3}{|c|}{ Nombroj de } & \multirow{3}{*}{$\begin{array}{l}\text { Pro- } \\
\text { centaj } \\
\text { Doma- } \\
\text { goj } P\end{array}$} & \multicolumn{3}{|c|}{ Nombroj de } & \multirow{3}{*}{$\begin{array}{l}\text { Pro- } \\
\text { centaj } \\
\text { Doma- } \\
\text { goj } P\end{array}$} \\
\hline & & \multicolumn{2}{|c|}{ Detruita 1} & \multirow[b]{2}{*}{$\underset{N}{\text { Tutaj }}$} & & \multicolumn{2}{|c|}{ Detruitaj } & \multirow{2}{*}{$\underset{N}{\text { Tutaj }}$} & \\
\hline & & $\begin{array}{c}\text { Tute } \\
N_{a}\end{array}$ & $\begin{array}{c}\text { Duone } \\
N_{\mu}\end{array}$ & & & $\begin{array}{l}\text { Tute } \\
N_{a}\end{array}$ & $\begin{array}{c}\text { Duone } \\
N_{h}\end{array}$ & & \\
\hline 1 & Higasi-mati & $1(1)$ & $14(15)$ & 54. & 14.9 & $8(11)$ & $14(24)$ & & 27.8 \\
\hline 2 & Nisi-ma & $4(4)$ & $35(39)$ & 6 & 34.8 & $4(4)$ & $18(23)$ & & 19.7 \\
\hline 3 & Naka-mati & $1(1)$ & $10(11)$ & 59 & 10.2 & $9(9)$ & $14(23)$ & & 27.1 \\
\hline 4 & Kami-mati-nisi & & $12(16)$ & 45 & 13.4 & $4(4)$ & $11(15)$ & & 21.1 \\
\hline 5 & $\begin{array}{l}\text { Kami-mati- } \\
\text { higasi }\end{array}$ & & $8(8)$ & 54 & & & $9(12)$ & & 8.3 \\
\hline 6 & Miya-no-uti & & $7(8)$ & 47 & 7.5 & $2(3)$ & $2(2)$ & & 6.4 \\
\hline 7 & Kita-mura & & & 10 & 0.0 & & & & $\begin{aligned} 10.0 \\
0.0\end{aligned}$ \\
\hline 8 & Tinzi & & $1(1)$ & 36 & 1.4 & & 2( & & 2.8 \\
\hline 9 & Naka-no-kawa & & & 63 & 0.0 & & $\left.\begin{array}{ll}6( & 6\end{array}\right)$ & & 1.8 \\
\hline 10 & Turi-no-kuti & & & 26 & 0.0 & (2) & $18(19)$ & & 3.0 \\
\hline 11. & a & & & $\begin{array}{l}369 \\
414\end{array}$ & 0. & & & & 0.0 \\
\hline & Sumo & $6(6)$ & $87(98)$ & 1243 & 4.0 & $29(33)$ & $97(130)$ & & 6.2 \\
\hline
\end{tabular}

de la urbeto trovighas iom da domaĝoj videblaj kompare al en la centra parto. Tamen en la 3-a kvartalo aperas jam influo de riverŝtonetoj kaj la domaĝoj estas iomete malpli severa. Domoj en la okcidenta parto estas sufice malfortikaj, sed tamen tio verŝajne estas kaŭzo de domâgoj tie, ĉar la kazo de la vilaĝo None montras la rilaton tute inversan kiel ni priskribos pli poste.

(2) Urbeto Kiragawa. Kiel montrate en Fig. 4, la vilaĝo kuŝas sur la montflanko proksimiĝanta al la maro kaj sur la sablajoj, kiuj de la mont-bazo etendighas al la direktoj orienta kaj okcikenta respektive. La rivero, kiu enfluas en la maron en la orienta elistremo de la vilagho, havas neniun aluvion. Tie roka bazo estas pli malprofunda kaj riverŝtonetaro pii granda ol en okciclenta limo.

Tertremaj domagoj estis severaj sur okcidenta sablajo kaj la parto sur la montflanko neniom suferis. (Vidu Tablon II kaj Fig 4.) $\mathrm{Cl}^{\prime}$-enhavo distribuighas kiel en Figr. 5 kaj la influo de subkusianta fluo de rivero estas granda en okcidenta parto. Sekve la rilato de Cl'-enhavo en la subterakvo al la tertremaj domấoj estas negativa, kaj la domagoj estas cefe regataj nur de la profundeco de roka bazo subtera. Sajnas probable, ke la sablajo de

Tabelo III. Domaĝoj en la vilaĝo None.

\begin{tabular}{|c|c|c|c|c|c|c|c|c|c|}
\hline \multirow{4}{*}{ No. } & \multirow{4}{*}{ Subvilaĝo } & \multicolumn{4}{|c|}{ Loĝdomoj } & \multicolumn{4}{|c|}{ Tenejoj, Stalo, k.t.p. } \\
\hline & & \multicolumn{3}{|c|}{ Nombroj de } & \multirow{3}{*}{$\begin{array}{l}\text { Pro- } \\
\text { centaj } \\
\text { Doma- } \\
\text { goj } P\end{array}$} & \multicolumn{3}{|c|}{ Nombroj de } & \multirow{3}{*}{$\begin{array}{l}\text { Pro- } \\
\text { centaj } \\
\text { Doma- } \\
\text { hoj } l^{\prime}\end{array}$} \\
\hline & & \multicolumn{2}{|c|}{ Detruitaj } & \multirow{2}{*}{$\underset{N}{\text { Tutaj }}$} & & \multicolumn{2}{|c|}{ Detivitaj } & \multirow{2}{*}{$\underset{N}{\text { Tutaj }}$} & \\
\hline & & $\begin{array}{c}\text { Tute } \\
N_{g}\end{array}$ & $\begin{array}{c}\text { Duone } \\
N_{h}\end{array}$ & & & $\begin{array}{c}\text { 'Tute } \\
N_{t}\end{array}$ & $\begin{array}{c}\text { Duone } \\
N_{h}\end{array}$ & & \\
\hline 1 & Ura-1-ku & 4 & 49 & 993 & 30.7 & 7 & 32 & & 23.7 \\
\hline$\frac{1}{2}$ & Ura-2-ku & 10 & 68 & 82 & 53.7 & 12 & 27 & & $2 \pi .8$ \\
\hline 3 & Ura-3-ku & 17 & 81 & 98 & 58.7 & 27 & 53 & & 51.0 \\
\hline 4 & Higasi-mati & 4 & 12 & 315 & 25.4 & 31 & 96 & & 24.6 \\
\hline 5 & Ike-no-aima & 8 & 23 & 49 & 39.8 & 17 & 38 & & 25.5 \\
\hline 6 & Nakamura & 35 & 45 & 83 & 72.0 & 55 & 83 & & 93.7 \\
\hline 7 & Nakazima & 3 & 11 & 44 & 19.3 & 2 & 8 & & 10.9 \\
\hline 8 & Kuzukago & 3 & 6 & 16 & 37.5 & 2 & 9 & & 31.4 \\
\hline 9 & Osino & 1 & 3 & 37 & 6.8 & & 2 & & 1.2 \\
\hline 10 & TItida & & 6 & 37 & 8.1 & & 2 & & 1.2 \\
\hline 11 & Oto & & 4 & 31 & 6.5 & & 3 & & 5.8 \\
\hline 12 & Kawaguti & & 3 & 36 & 4.2 & & 2 & & 1.4 \\
\hline 13 & Masunase & & 3 & 32 & 4.7 & . & 1 & & 0.9 \\
\hline 14. & Betuyaku & & 2 & 32 & 3.2 & & $\hat{1}$ & & 0.8 \\
\hline \multirow{2}{*}{15} & Natome-gawa & 4 & 46 & 108 & 25.0 & 8 & 21 & & 7.5 \\
\hline & Sumo & 99 & 482 & 1080 & 31.4 & 151 & 878 & & 20.2 \\
\hline
\end{tabular}


la okcidenta parto de la vilaĝo konsistis el blovita sablo de pli marproksima tereno, kaj konsekvence la severaj domaĝjoj tie farighas klaraj.

(3) Vilaĝo None. Distribuo en ciuj subvilaĝoj estas montrata en Tabelo III. Subvilaĝo Nakamura (N-ro 6), kiu kuŝas meze de la aluvio, suferis la plej severajn domaĝjojn kaj Ura-2, Ura-3, sur la marborda digo sekvis

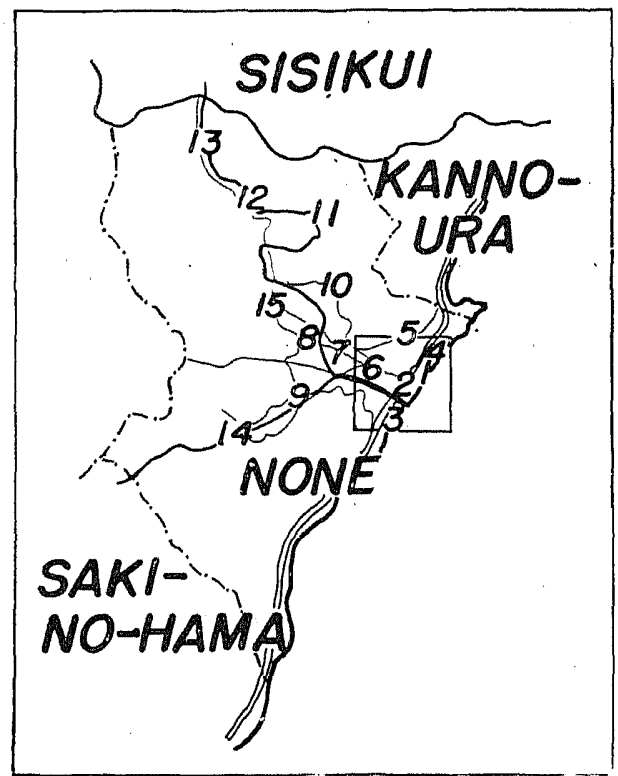

Fig. 6. Indeksa Landkarto de la subvilaĝoj en la Vilaĝo None, Koti Prefektejo.

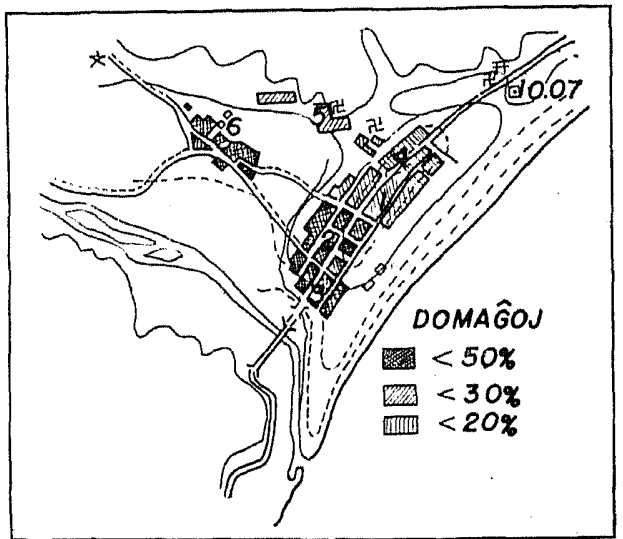

Fig. 7. Domaĝoj de la Nankaido Tertremo en la vilaĝo None, Koti Prefektejo.

gin en domấgoj. Estas malgrandaj domaĝjoj ankaŭ en Ura-1, Higasimati, Ike-no-aima,
Nakazima, kaj Kuzukago; la unuaj du situacias sur la marborda digo de sabla terajo kaj la lastaj tri estas ĉirkaŭ $\mathrm{kaj}$ en la aluvia ebenejo. (Vidu Figurojn $6 \mathrm{kaj}$ 7.)

Kiel ci supre skribite, la domaĝoj en Nakamura estas tre grandaj. Surfaca terajo tie konsistas el komplekso de argilo kaj ŝtonetoj, kaj tio montras, ke gi estas riveramaso. Sed laŭ geomorfologia vidpunkto ni povas konjekti, ke subtere estas malgrandaj grajnetoj de argilo kaj sekve la tereno estis iam laguno malanataŭ la marborda digo.

Montflanko cirkaŭ la aluvio estas tre kruta kaj tio montras la grandan dikecon de la aluvio. La lastaj konjektoj probable klarigas kaŭzon de la severeco de la tertremaj domaĝoj tie.

Sur la marbordo de la sud-okcidenta parto (Ura-2 kaj Ura 3) suferas severe de la tertremo, kaj en la nord-orienta parto (Ura-1 kaj Higasimati) la domậoj estas malgrandaj. (Komparu la paragrafon pri Aki urbeto:)

$\mathrm{Cl}$-enhavo de put-akvo en la vilaĝo estas generale tre malmulta kaj influighas iomete de irigacita rizokampo en okcidento kaj svarmado de domaĉj en nord-orienta parto. En nord-orienta parto ni povas supozi moviĝadon de subterakvo. (Vidu Fig. 8)

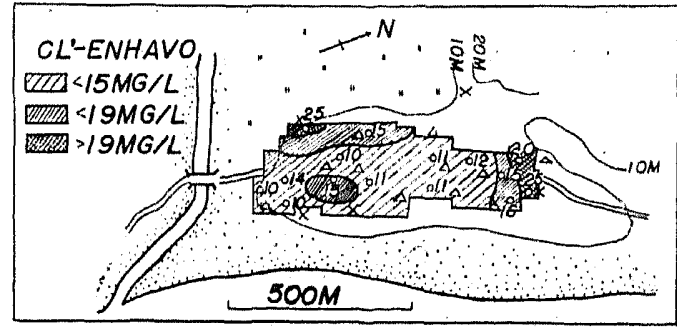

Fig. 8. Cl'-enhavo en subterakvo kaj rezulto de borbastona ekzameno en la vilaĝo None, Koti Prefektejo. ( $x$ mola $\triangle$ malmola)

Laŭ la borbastona ekzameno, surfaca teraĵo tie troviĝis mola cirkonference de la marborda digo. Denaske la marborda digo kreskis de la nord-oriento kiej. bekforma sablajo laŭ nia konjekto, tial la distribuo de domấoj klarighas de noveco de naskiĝo de la sablajo. Ankaŭ sub la nord-orienta parto estas roka bazo malprofunda. 
II. Riverregiono de la Rivero Mononobe kaj la ĉirkaŭajo de la Urbo Koti.

La valo de la supra parto de la rivero Mononobe estas relative mallarĝa sur si kaj en diversaj lokoj trovighas riverterasoj kun maldika amaso. Tertremaj domậoj estas tie malgrandaj.

La orienta parto de Koti-ebenejo estas okupata de ventumilforma tereno de malsupra parto de R. Mononobe kaj trovighas tie nemalmultaj urbetoj kaj vilaĝoj. Tertremaj domaĝoj sendube estas malgrandaj, car ĝenerale la ventumilforma tereno estas ŝtonetplena kaj rezistas forte al tertremo. Kontraŭ tio, la okcidenta parto, cirkaŭajo de la urbo Koti konsistas el aluviaj ebenejoj, kiuj malaltiĝas inter dislokîgoj, kurantaj de oriento al okcidento, tereno tie estas malalta $\mathrm{kaj}$ malseka $\mathrm{kaj}$ oni trovas sufice severajn tertremajn domaĝojn.

Partoj de la urbo Koti, kiuj estas malfortaj al tertremo, estis plene forbruligita de usona aeratako kaj tial procentaj domấoj de la tertremo estas tre malgrandaj malgraŭ supozo. Sekve, por tiu ĉi distrikto estas necesa singardo.

Marborda distrikto de Koti-ebenejo konsistas el marborda digo kaj malalta $\mathrm{kaj}$ malseka tereno malantaŭ $\hat{g} i$. Ekzemple en la urbetoj Kisimoto, Miwa kaj aliaj trovighas severaj domaĝjoj sur $\hat{c} i$ tiuj malaltaj terenoj malantaŭ la digoj.

\section{Riverregiono de la $R$. Yosino.}

La supra parto de "antecedanta" rivero Yosino ne havas larĝan valon, kaj sekve trovighas malmultaj vilaĝoj en la regiono. Tertremaj domaĝoj ne ekzistas tie, kaŭze de solida grund-naturo.

IV. Riverregiono de la R. Niyodo.

En la supra parto troviĝas malgrandaj domaĝjoj de la tertremo, sed la malsupra parto suferas iom severe kaj estas pli dangere tie ol la riverregiono de la $\mathrm{R}$. Mononobe.

$V$. Regiono de la supra parto de $R$. Simando.

Meza parto de R. Simando meandras enmetate konsekvence de lasta ter-leviĝo kaj havas riverterason, sur kiu estas dislokitaj urbetoj kaj vilaĝoj. Ne trovighas tre severaj tertremaj domaĝj, krom en urbeto Kubokawa, kie procentaj domaĝjoj superas 1\%. Tamen la lasta urbeto situacias sur iom larĝa inunda aluvio, kiu naskiĝis pro tio, ke nur tiu parto mallevighis en plej nova geologia epoko kontraŭ pli malsupra parto, kiu leviĝis samtempe.

VI. Riverregiono de la malsupra parto de R. Simando, Nakasuzi Terfosajo k.a.

En la centro de la regiono situacias la urbeto Nakamura, kiu suferis la plej severajn domaĝjojn de Nankaido-tertremo kiel jam skribite. La malsupra parto de la R. Simando nomighas ankaŭ $R$. Watari, kaj ĝi fluas tie ankaŭ kun iom kruta kliniĝo. Kontraŭe ĝiaj enfluantoj Nakasuzi kaj Usiro havas tre malkrutajn riverujojn. Precipe la lito de $R$. Nakasuzi estas pli malalta ol tiu de R. Watari (ĉefa fluo) ĉe la enfluejo, kaj ĝis $8.5 \mathrm{~km}$ supra punkto ĝ ia fluosuprajo restas horizonta. Tial la supra riverregiono de $\mathrm{R}$. Watari estas tre vasta kaj okaze cle inundo ekfluadas grandega kvanto da akvo, parto de akvo enfluas tiam kontraŭflue en la fluejon de R. Nakasuzi kaj $R$. Usiro ofte okazigante superakvegon sur la cirirkaŭajo.

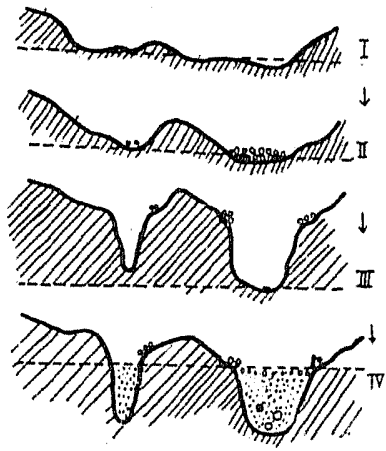

Fig. 9. Geomorfologia Historio cirkaŭ Nakamura, kantono de Hata, Koti Prefektejo.

La terfosajo de Nakasuzi (germane Nakasuzi-graben) naskigi is post la kreta periodo, sed la diluvia tavolo tie suferas nenian agitiĝon, kaj tio montras, ke la dislokjĝoj ne movighas post la kvarta periodo. Tial oni ne: povas rilatigi la dislokiĝjn kun nuna ter- 
tremo, malgraŭ asertoj de kelkaj aŭtoroj.

Ebenejo en tiu distrikto estas tre dika aluvio, kies amaso konsistas el ślimo el mal:grandegaj eroj, kaj eĉ tre proksime de monto roka bazo estas tiel profunda, kiel $50 \mathrm{~m}$ borado ne povas atingi ĝin. La geomorfologia historio de la regiono povas doni bonan klarigon al tio. Nome, kiel montrate en Fig. 9, unue naskiĝis larĝaj peronoj kaŭzitaj de mar- aŭ river-erozio, due ili levighis kaj formis terasojn, ekzemple la malaltan terason norde de urbeto Simoda, trie ili plie leviốis intermitante $\mathrm{kaj}$ formas kelkajn stupojn de terasoj (ekzemple marterasoj kiuj subportas subvilaĝon Hirano, urbeton Simoda, aŭ subvilaĝon Iya, vilaĝon Taguti kaj riverterason, sur kiuj restas subvilaĝo Taguro, vilaĝo Gudo.) Leviĝ́oj de ĉi tiu periodo estas sufice grandaj, la erozio daŭras longe, kaj formas profundajn valojn, kun tre krutaj flankoj. Kvare, tiam la tereno mallevigis $\mathrm{kaj}$ maro invadis en la valojn, kie akumligis dika aluvia tavolo ĝis la marsuprajo. Post tiam R. Simando multigis sian transportan forton, kaŭzis leviĝon en sia mez-flua regiono, kaj portis stonetojn, kiuj kovris maldike la suprajon de la aluvio. (Vidu priskribon pri la urbeto Nakamura.)

La baza roko en tiu distrikto estas alternaj tavoloj de sabloŝtonoj kaj skistargilo. Malgraŭ ke la kialo estas kompreneble nekonataj partoj de skistargilo detruiĝas tre facile pro efloresko. Tio alportas tertremajn domaĝojn pro terfalajo en la regiono de Nakasuzi kaj ankaŭ havus iom da influo al ĝenerala severeco de domaĝoj en la tuta distrikto.

Proksime al montaro troviĝas tie ankaŭ konsiderindaj domaĝoj de la tertremo malgraŭ generalaj ekzemploj. Krom la supre citita naturo de roka bazo, tio devenas de artefarita amasigo, kiu estas populara en tiu regiono aŭ por akiri larĝan konstruan terenon ce la kruta montflanko aŭ por eviti superakvon de oftaj inundoj.

Kruteco de valformo estas klara per sisma ter-esploro de d-ro K. KaNAI kaj ekzameno de putoj proksime de montflanko.

(1) Urbeto Nakamura. Pri la distribuo de tertremaj domaĝoj en la urbeto d-ro Kanai jam aliloke skribis. ${ }^{1)}$ Nu sekvos ĉi-sube kelkaj

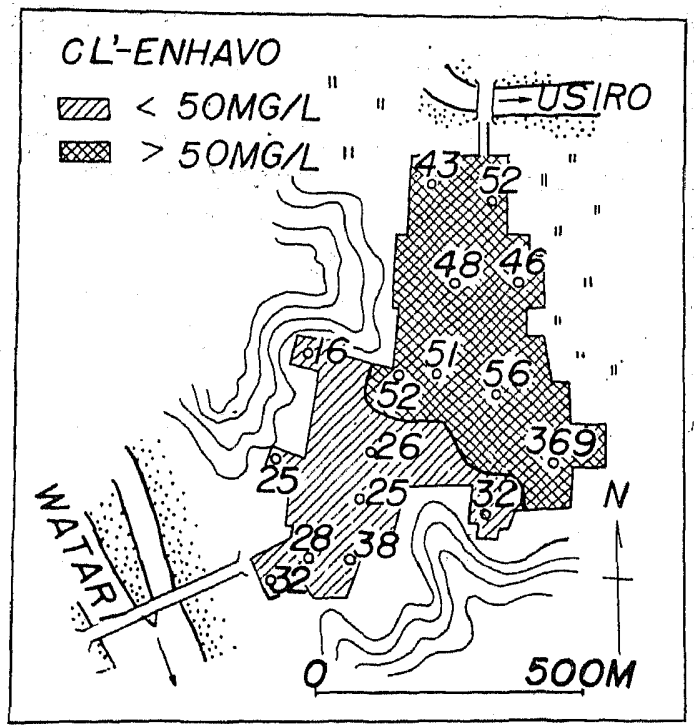

Fig. 10. Cl'-enhavo en subterakvo sub la urbeto Nakamura, Koti Prefektejo.

rimarkoj pri interrilatoj de domaĝoj kaj la naturoj de grundo.

En la orienta duono de la urbeto suferas pli severe de la tertremo kaj tio bone respondas al la distribuo de richa $\mathrm{Cl}^{\prime}$-enhavo en subtera akvo kiel montrate en. Fig. 10.

La subtera akvo en la okcidenta parto enhavas pli malmulte da klor-jono ol en la orienta parto, car la unua fluadas tra la stonetplena akvoportanta tavolo amasigita de R. Simando kaj la lasta restas, kontraŭe, en la slima subtera tavolo amasigita de R. Usiro, kaj tiu malsameco inter du partoj donas sendube supre priskribitan distribuon de tertremaj domaĝoj. Kiel aliaj aŭtoroj skribis, ĉiuj domoj en la urbetoj konstruiĝis sur amasigataj terenoj por bari la oftajn superakvegojn.

VIII. Marborda distrikto de sud-okcidenta parto de la kantono Hata.

La distrikto enhavas marbordon de cirlkaŭajo de Suzaki, preter promontoro Asizuri gis la urbeto Sukumo. Kvankam en la norda parto ne trovighas marborda teraso, oni trovas ĝin de cirkaŭ Saga kaj, malsame de kazo de Muroto duoninsulo, vilaĝoj restas sur git. Kompreneble estas ankaŭ vilaĝoj sur marborda digo kaj malsupro de monto pro- 
ksimighas al la maro. Sube sekvos rezultoj de esploroj en la vilaĝoj Ōgata, Simokawaguti kaj la urbeto Sukumo.

(1) Vilâgo Ōgata. Nur en la subvilaĝo Irino ni faris niajn esplorojn. La tereno estas malalta sutplenigita sablejo inter marbordo malproksima sablajo kaj teraso kaj suferas treege de la tertremo. Kiel montrate en Fig. 11, domấoj estis severaj en Hayasaki (1), Siba (6), Mangyo (5) kaj Mati (3). Aliflanke la domaĝj estis iom malseveraj en Honmura (4)

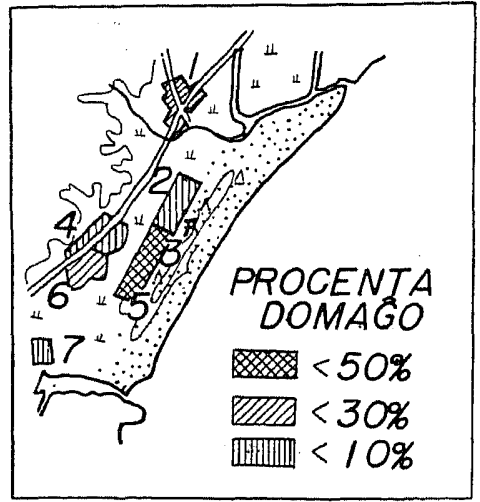

Fig. 11. Domaĝjoj de la Nankaido Tertremo en Irino, vilaĝo Ogata, Koti Prefektejo.

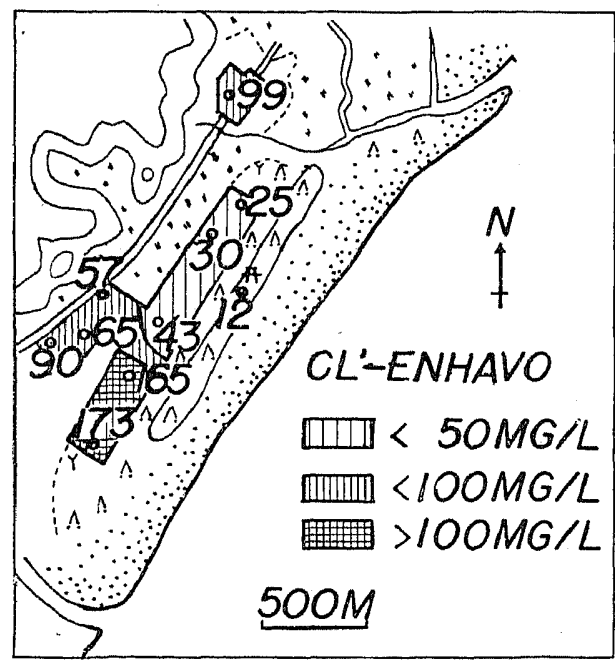

Fig. 12. Cl'-enhavo en subterakvo en la subvilaĝo Irino, vilaĝo Ōgata, Koti Prefektejo.

proksima al malsupro de teraso $\mathrm{kaj}$ en Hamanomiya (2), kiu situacias sur la sablejo de marborda digo. Cl'-enhavo en la subtera akvo distribuighas tie kiel en Fig. 12, kie klare montrighas la parto kun staranta subterakvo. Granda Cl'-enhavo en Mangyo devenas ankaŭ de malpura stato de domaro.

(2) Vilaĝ́o Simokawaguti. Tertremaj domaĝoj estas montrataj en Fig. 13, Severaj domaĝoj distribuighas en la malalta tereno malantaŭ sablajo kaj en la malantaŭa flanko de la sablaĵo. Kontraŭe sur la sablajo mem precipe en la proksima parto de roka bazo sin trovas neniu domaĝo.

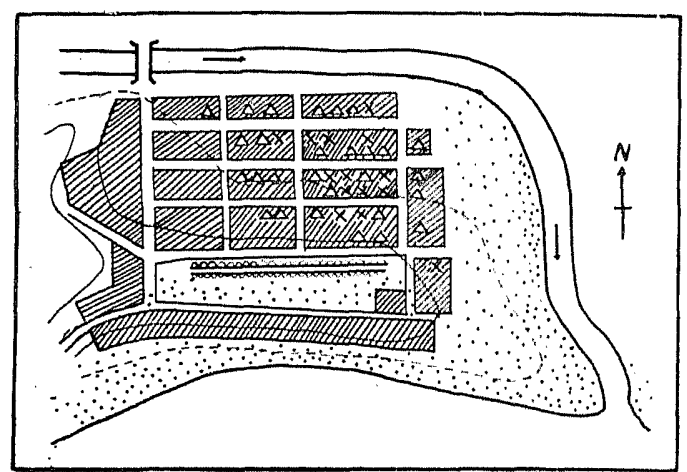

Fig. 13. Domaĝoj de la Nankaido Tertremo en la vilaĝo Simokawaguti, Koti Prefektejo. ( $\times$ tute $\triangle$ duone)

Ciuj supraj respondas al la distribuo de $\mathrm{Cl}$ enhavo en la subterakvo, montrata en Fig. 14. Oni ne povas rimarki subteran fluon influatan de proksima rivero. La valoroj de Cl'-enhavo mem estas tre grandaj, sed la kialo estas neklara.

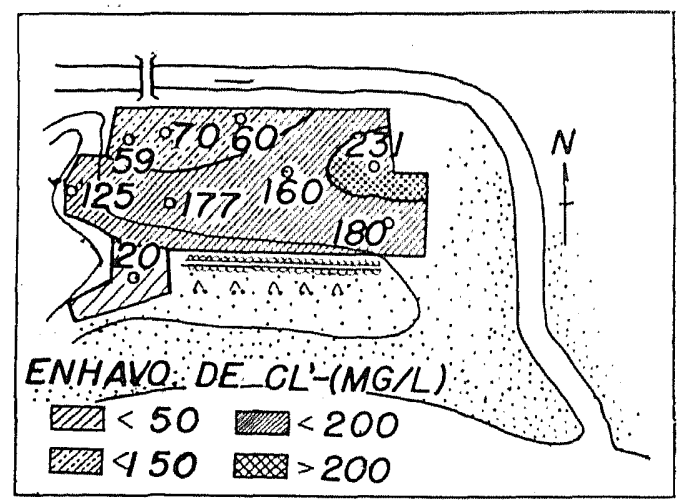

Fig. 14. Cl'-enhavo en subtera akvo en la vilâgo de Simokawaguti, Koti Prefektejo. 


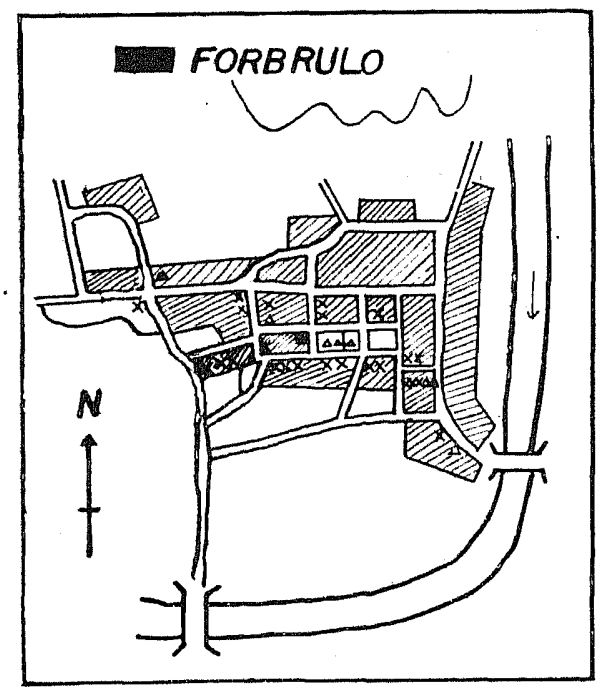

Fig. 15. Domaĝoj de la Nankaido Tertremo en la urbeto Sukumo, Koti Prefektejo. ( $\times$ tuta detruiĝo $\Delta$ duona detruiĝo)

(3) Urbeto Sukumo. Sukumo estas interlanda urbeto kaj ne marborda. Kvankam la subterakva Cl'-enhavo klare dividas la urbeton en du partojn tute same kiel en la urbeto Nakamura, tamen estas ne tiel klara rilato al la tertremaj domaĝoj en tiu ĉi okazo. (Vidu Fig. 15) La domaĝjoj estas regataj cefe de la dikeco de aluvio, kiu farighas iom post iom maldika proksimighante al la monto.

Fine la aŭtoroj deziras esprimi siajn korajn dankojn al la loĝantoj kaj la aŭtoritatularo

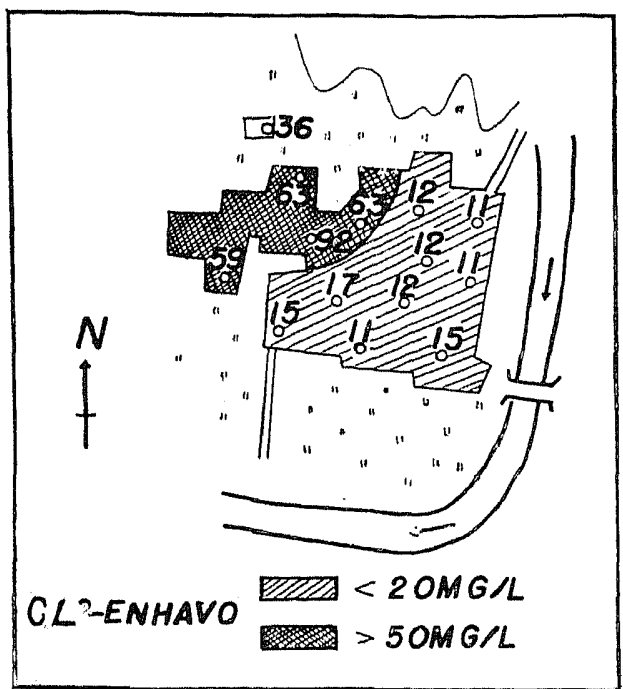

Fig. 16. Cl'-enhavo en subterakro en la urbeto Sukumo, Koti Prefektejo.

cie en Koti Prefektejo, kiuj bonvole helpis iliajn esplorojn. Ankaŭ dankon al d-ro K. KanaI de la Instituto por Esploroj de Tertremo por lia bonkora rilatigo al la estraro de la prefektejo. S-rinoj K. Noumoto kaj S. Kondô helpis la aŭtorojn en preparado de l'figuroj.

\section{Referenco}

1) K. KANAI, Zisin Kenkyu-syo Kenkyusokuhô No. 5 (1947, IV) (En Japana lingvo) 